\title{
A DEFECT RELATION FOR NON-ARCHIMEDEAN ANALYTIC CURVES IN ARBITRARY PROJECTIVE VARIETIES
}

\author{
TA THI HOAI AN
}

(Communicated by Ken Ono)

\begin{abstract}
If $f$ is a non-Archimedean analytic curve in a projective variety $X$ embedded in $\mathbb{P}^{N}$ and if $D_{1}, \ldots, D_{q}$ are hypersurfaces of $\mathbb{P}^{N}$ in general position with $X$, then we prove the defect relation:
\end{abstract}

$$
\sum_{j=1}^{q} \delta\left(f, D_{j}\right) \leq \operatorname{dim} X .
$$

\section{INTRODUCTION}

Nevanlinna theory is a generalization of the fundamental theorem of algebra to meromorphic functions over the complex numbers, or more generally to meromorphic maps between complex spaces. In the past few decades, there has been interest in a non-Archimedean Nevanlinna theory; cf. [HY].

Classical Nevanlinna theory for meromorphic functions over the complex numbers consists of two fundamental inequalities. The First Main Theorem, which is a reformulation of the Poisson-Jensen formula, provides an upper bound on the frequency with which a meromorphic function can take on a complex value in terms of the growth order of the function. The deeper Second Main Theorem provides a lower bound. At the same time, the Second Main Theorem gives an upper bound on the ramification, i.e., the frequency with which the function can take on values with multiplicity greater than one. The First Main Theorem can easily be extended to the general case of meromorphic mappings between complex spaces. However, analogs of the Second Main Theorem remain largely conjectures when the target is something other than a projective space or a semi-Abelian variety.

In $[\underline{\mathrm{R}}$, Ru observed that in stark contrast to the situation over the complex numbers, over non-Archimedean ground fields, if one drops the ramification term from the Second Main Theorem, then it follows from the First Main Theorem. Using this observation, Ru gave a simple proof of an inequality of Second Main Theorem type, without ramification, for curvilinear hypersurfaces in general position in projective space. Note that $\mathrm{Ru}$ states his results using the concrete ground field $\mathbb{C}_{p}$, the $p$-adic complex numbers.

Received by the editors November 21, 2005 and, in revised form, November 28, 2005.

2000 Mathematics Subject Classification. Primary 12E05, 11S80, 30D25.

Financial support provided to the author as a Junior Associate by ICTP, Trieste, Italy. 
In this note, we extend Ru's result to arbitrary subvarieties of projective space and remark that the argument works over any algebraically closed field complete with respect to a non-Archimedean absolute value, even in positive characteristic.

\section{Notation And STATEMEnt of Results}

Let $\mathbf{K}$ be an algebraically closed field of arbitrary characteristic, complete with respect to a non-Archimedean absolute value | |. If

$$
f(z)=\sum_{j=0}^{\infty} a_{n} z^{n}
$$

is an entire function on $\mathbf{K}$, then for each real number $r \geq 0$, we define

$$
\begin{aligned}
|f|_{r} & =\sup _{j}\left|a_{j}\right| r^{j}=\sup \{|f(z)|: z \in \mathbf{K} \text { with }|z| \leq r\} \\
& =\sup \{|f(z)|: z \in \mathbf{K} \text { with }|z|=r\} .
\end{aligned}
$$

Let $f: \mathbf{K} \rightarrow \mathbb{P}^{N}(\mathbf{K})$ be a non-constant analytic curve in projective space. Let $\tilde{f}=$ $\left(f_{0}, \ldots, f_{N}\right)$ be a reduced representative of $f$, where $f_{0}, \ldots, f_{N}$ are entire functions on $\mathbf{K}$ without common zeros, at least one of which is non-constant.

The Nevanlinna characteristic function $T_{f}(r)$ is defined by $T_{f}(r)=\log \|f\|_{r}$, where

$$
\|f\|_{r}=\max \left\{\left|f_{0}\right|_{r}, \ldots,\left|f_{N}\right|_{r}\right\} .
$$

The above definition of $T_{f}(r)$ is independent, up to an additive constant, of the choice of the reduced representation of $f$.

Let $D$ be a hypersurface in $\mathbb{P}^{N}(\mathbf{K})$ of degree $d$. Let $Q$ be the homogeneous polynomial (form) in $N+1$ variables with coefficients in $\mathbf{K}$ defining $D$. We consider the entire function $Q \circ f=Q\left(f_{0}, \ldots, f_{N}\right)$ on $\mathbf{K}$. Let $n_{f}(r, Q)$ be the number of zeros of $Q \circ f$ in the (closed) disk $\mathbf{B}[r]=\{z \in \mathbf{K}:|z| \leq r\}$, counting multiplicity. If $Q \circ f \not \equiv 0$, set

$$
\begin{aligned}
& N_{f}(r, D)=N_{f}(r, Q)=\int_{0}^{r} \frac{n_{f}(t, Q)-n_{f}(0, Q)}{t} d t+n_{f}(0, Q) \log r, \\
& m_{f}(r, D)=m_{f}(r, Q)=\log \frac{\|f\|_{r}^{d}}{|Q \circ f|_{r}} .
\end{aligned}
$$

Note that up to a constant term, $m_{f}(r, D)$ is independent of the choice of the defining form $Q$. The functions $N_{f}(r, D)$ and $m_{f}(r, D)$ are referred to as the counting and proximity functions, respectively.

A collection of $q \geq N+1$ hypersurfaces $D_{1}, \ldots, D_{q}$ with associated defining forms $Q_{1}, \ldots, Q_{q}$, is said to be in general position in $\mathbb{P}^{N}(\mathbf{K})$ if for any subset $\left\{i_{0}, \ldots, i_{N}\right\}$ of $\{1, \ldots, q\}$ of cardinality $N+1,\left\{x \in \mathbb{P}^{N}(\mathbf{K}): Q_{i_{j}}(x)=0, j=0, \ldots, N\right\}=\emptyset$. $\mathrm{Ru}[\mathrm{R}]$ proved the following.

Ru's Theorem. Let $D_{1}, \ldots, D_{q}$ be hypersurfaces of $\mathbb{P}^{N}(\mathbf{K})$ in general position. Let $f$ be a non-constant analytic curve from $\mathbf{K}$ to $\mathbb{P}^{N}(\mathbf{K})$ whose image is not completely contained in any of the hypersurfaces $D_{1}, \ldots, D_{q}$. Then for any positive real number $r$,

$$
\sum_{j=1}^{q} \frac{m_{f}\left(r, D_{j}\right)}{\operatorname{deg} D_{j}} \leq N T_{f}(r)+O(1)
$$

where $O(1)$ is a constant independent of $r$. 
In this note, we show that if the image of $f$ is contained in an $n$-dimensional subvariety $X$, then the $N$ on the right-hand side of the inequality in Ru's theorem can be replaced by the dimension of $X$. Before stating the theorem, we need to generalize the definition of "in general position".

Let $X$ be an $n$-dimensional (not necessarily smooth) projective subvariety of $\mathbb{P}^{N}(\mathbf{K})$. A collection of $q \geq n+1$ hypersurfaces $D_{1}, \ldots, D_{q}$ in $\mathbb{P}^{N}(\mathbf{K})$ is said to be in general position with $X$ if for any subset $\left\{i_{0}, \ldots, i_{n}\right\}$ of $\{1, \ldots, q\}$ of cardinality $n+1,\left\{x \in X: Q_{i_{j}}(x)=0, j=0, \ldots, n\right\}=\emptyset$.

Main Theorem. Let $X \subset \mathbb{P}^{N}(\mathbf{K})$ be a projective subvariety of dimension $n \geq 1$ over $\mathbf{K}$. Let $D_{1}, \ldots, D_{q}$ be hypersurfaces in $\mathbb{P}^{N}(\mathbf{K})$ in general position with $X$. Let $f: \mathbf{K} \rightarrow X$ be a non-constant analytic map whose image is not completely contained in any of the hypersurfaces $D_{1}, \ldots, D_{q}$. Then, for any positive real number $r$,

$$
\sum_{j=1}^{q} \frac{m_{f}\left(r, D_{j}\right)}{\operatorname{deg} D_{j}} \leq n T_{f}(r)+O(1),
$$

where $O(1)$ is a constant independent of $r$.

We remark that the hypersurfaces $D_{j}$ are hypersurfaces of the ambient projective space $\mathbb{P}^{N}(\mathbf{K})$ and not arbitrary effective divisors of $X$. In more intrinsic terms, we are essentially considering very ample effective divisors in general position on $X$.

We note that Evertse and Ferretti $[\mathrm{EF}$ ] have proven a height inequality that is the arithmetic analog of the inequality in our Main Theorem here.

Define the defect $\delta_{f}\left(D_{j}\right)$ of the hypersurface $D_{j}$ with respect to the map $f$ by

$$
\delta_{f}\left(D_{j}\right)=\liminf _{r \rightarrow \infty} \frac{m_{f}\left(r, D_{j}\right)}{\left(\operatorname{deg} D_{j}\right) T_{f}(r)} .
$$

With the defect notation, our Main Theorem immediately results in the following corollary.

Corollary 1. With notation and assumptions as in the theorem,

$$
\sum_{j=1}^{q} \delta_{f}\left(D_{j}\right) \leq n=\operatorname{dim} X
$$

William Cherry has suggested to us that we can use the idea of [CY, Theorem 6.1] to strengthen this to

Corollary 2. With the assumptions and notation as in the theorem, the number of hypersurfaces $D_{j}$ such that $\delta_{f}\left(D_{j}\right)>0$ is at most $n$.

\section{Proof of the Main Theorem}

We first recall the Nevanlinna First Main Theorem in non-Archimedean fields (cf. $[\mathrm{R}]$ ).

First Main Theorem. Let $f: \mathbf{K} \rightarrow \mathbb{P}^{N}(\mathbf{K})$ be an analytic curve and let $Q$ be a homogeneous form of degree $d$. If $Q(f) \not \equiv 0$, then for every positive real number $r$,

$$
m_{f}(r, Q)+N_{f}(r, Q)=d T_{f}(r)+O(1),
$$

where $O(1)$ is a constant independent of $r$. 
Proof of the Main Theorem. For $i=1, \ldots, q$, let $Q_{i}$ be the homogeneous form in $\mathbf{K}\left[x_{0}, \ldots, x_{N}\right]$ of degree $d_{i}$ defining the hypersurface $D_{i}$. Let $\Delta$ be the least common multiple of the $d_{i}$ 's. Then $G_{i}:=Q_{i}^{\Delta / d_{i}}$ are homogeneous polynomials of the same degree $\Delta$.

For fixed $r$, by rearranging the indices if necessary, we may assume that

$$
\left|G_{1} \circ f\right|_{r} \leq\left|G_{2} \circ f\right|_{r} \leq \cdots \leq\left|G_{q} \circ f\right|_{r} .
$$

Since $Q_{1}, \ldots, Q_{q}$ are in general position with $X$ by assumption, $G_{1}, \ldots, G_{q}$ are also in general position with $X$. Therefore,

$$
X \cap\left\{G_{1}=0, \ldots, G_{n+1}=0\right\}=\emptyset .
$$

Applying Hilbert's Nullstellensatz (cf. [W]) to the ideal generated by the forms defining $X$ and $G_{1}, \ldots, G_{n+1}$, we see that for any integer $k, 0 \leq k \leq N$, there is an integer $m_{k} \geq \Delta$ such that

$$
x_{k}^{m_{k}}=\sum_{i=1}^{n+1} b_{i k}\left(x_{0}, \ldots, x_{N}\right) G_{i}\left(x_{0}, \ldots, x_{N}\right) \quad \text { on } X,
$$

where $b_{i k}$, for $1 \leq i \leq N, 0 \leq k \leq N$, are homogeneous forms with coefficients in $\mathbf{K}$ of degree $m_{k}-\Delta$. Hence

$$
\left|f_{k}\right|_{r}^{m_{k}} \leq\left. C|| f\right|_{r} ^{m_{k}-\Delta} \max \left\{\left|G_{1} \circ f\right|_{r}, \ldots,\left|G_{n+1} \circ f\right|_{r}\right\},
$$

where $C$ is a positive constant that depends only on the coefficients of the $b_{i k}$. Therefore,

$$
\|f\|_{r}^{\Delta} \leq C \max \left\{\left|G_{1} \circ f\right|_{r}, \ldots,\left|G_{n+1} \circ f\right|_{r}\right\} .
$$

Thus, continuing to keep $r$ fixed,

$$
\begin{aligned}
\Delta \sum_{j=1}^{q} \frac{m_{f}\left(r, Q_{j}\right)}{\operatorname{deg} Q_{j}}=\sum_{j=1}^{q} m_{f}\left(r, G_{j}\right) & =\sum_{j=1}^{q} \log \frac{\|f\|_{r}^{\Delta}}{\left|G_{j} \circ f\right|_{r}} \\
& =\sum_{j=1}^{n} \log \frac{\|f\|_{r}^{\Delta}}{\left|G_{j} \circ f\right|_{r}}+\sum_{j=n+1}^{q} \frac{\|f\|_{r}^{\Delta}}{\left|G_{j} \circ f\right|_{r}} \\
\text { [from (2) and (1) }] & \leq \sum_{j=1}^{n} \log \frac{\|f\|_{r}^{\Delta}}{\left|G_{j} \circ f\right|_{r}}+O(1) \\
& =\sum_{j=1}^{n} m_{f}\left(r, G_{j}\right)+O(1)
\end{aligned}
$$

[by the First Main Theorem] $\leq n \Delta T_{f}(r)+O(1)$.

Because the right-hand side of the inequality no longer depends on the arrangement of the indices in (1) and the $O(1)$ term is independent of $r$, dividing both sides through by $\Delta$ gives the theorem for all positive $r$.

Proof of Corollary 2. If the corollary were not true, then $n+1$ of the hypersurfaces could have a non-zero defect. Thus, without loss of generality, we may suppose $\delta_{f}\left(D_{j}\right)>0$ for $j=1,2, \ldots, n+1$. By definition, that means there exists $\varepsilon>0$ such that for all $r$ sufficiently large,

$$
m_{f}\left(r, D_{j}\right)>\varepsilon\left(\operatorname{deg} D_{j}\right) T_{f}(r) \quad \text { for } j=1, \ldots, n+1 .
$$


Letting $G_{j}$ be as in the proof of our Main Theorem, we then have for all $r$ sufficiently large,

$$
m_{f}\left(r, G_{j}\right)>\varepsilon \Delta T_{f}(r) \text { for } j=1, \ldots, n+1 .
$$

Hence, for all $r$ sufficiently large and for $j=1, \ldots, n+1$,

$$
\left|G_{j} \circ f\right|_{r}<\|f\|_{r}^{\Delta(1-\varepsilon)} .
$$

Because $f$ is non-constant, this contradicts (2) by applying the Nullstellensatz argument of the previous proof to $G_{1}, \ldots, G_{n+1}$.

\section{TWO VARIATIONS}

Using the slicing technique of [CY], we immediately get

Theorem 3. Let $X \subset \mathbb{P}^{N}(\mathbf{K})$ be a projective subvariety of dimension $n \geq 1$ over $\mathbf{K}$. Let $D_{1}, \ldots, D_{q}$ be hypersurfaces in $\mathbb{P}^{N}(\mathbf{K})$ in general position with $X$. Let $f: \mathbf{K}^{m} \rightarrow X$ be a non-constant analytic map whose image is not completely contained in any of the hypersurfaces $D_{1}, \ldots, D_{q}$. Then, for any positive real number $r$

$$
\sum_{j=1}^{q} \frac{m_{f}\left(r, D_{j}\right)}{\operatorname{deg} D_{j}} \leq n T_{f}(r)+O(1),
$$

where $O(1)$ is a constant independent of $r$.

In $[$ ], Cherry found it interesting to look at maps from $\mathbf{K} \backslash\{0\}$, rather than from all of $\mathbf{K}$. An analytic function $f(z)$ on $\mathbf{K} \backslash\{0\}$ is represented by a Laurent series

$$
\sum_{j=-\infty}^{\infty} a_{j} z^{j}
$$

and for $r>0$, we define

$$
|f|_{r}=\sup _{j}\left|a_{j}\right| r^{j}=\sup \{|f(z)|: z \in \mathbf{K} \text { with }|z|=r\} .
$$

A non-constant analytic curve $f: \mathbf{K} \backslash\{0\} \rightarrow \mathbb{P}^{N}(\mathbf{K})$ is defined by a reduced representation $\left(f_{0}, \ldots, f_{N}\right)$, where $f_{j}$ are analytic on $\mathbf{K} \backslash\{0\}$, and where at least one of the $f_{j}$ is not constant. As before, set

$$
\|f\|_{r}=\max \left\{\left|f_{0}\right|_{r}, \ldots,\left|f_{N}\right|_{r}\right\} .
$$

However, note this time that changing the reduced representation of $f$ will change $\|f\|_{r}$ possibly by a power of $r$ and a multiplicative constant.

For the Nevanlinna functions, we only consider $r \geq 1$. For the characteristic function, set

$$
T_{f}(r)=\log \|f\|_{r}+\log \|f\|_{r^{-1}}-2 \log \|f\|_{1},
$$

which is independent of the choice of reduced representation for $f$. For $Q$ a homogeneous form defining a hypersurface $D$ of degree $d$, define the counting function by

$$
N_{f}(r, D)=N_{f}(r, Q)=\sum_{r^{-1} \leq|z| \leq r} \operatorname{ord}_{z}(Q \circ f)(\log r-|\log z|),
$$

and the proximity function by

$$
m_{f}(r, D)=m_{f}(r, Q)=\log \frac{\|f\|_{r}^{d}}{|Q \circ f|_{r}}+\log \frac{\|f\|_{r-1}^{d}}{|Q \circ f|_{r^{-1}}}-2 \log \frac{\|f\|_{1}^{d}}{|Q \circ f|_{1}} .
$$


Theorem 4. Let $X \subset \mathbb{P}^{N}(\mathbf{K})$ be a projective subvariety of dimension $n \geq 1$ over K. Let $D_{1}, \ldots, D_{q}$ be hypersurfaces in $\mathbb{P}^{N}(\mathbf{K})$ in general position with $X$. Let $f: \mathbf{K} \backslash\{0\} \rightarrow X$ be a non-constant analytic map whose image is not completely contained in any of the hypersurfaces $D_{1}, \ldots, D_{q}$. Then, for any real number $r>1$,

$$
\sum_{j=1}^{q} \frac{m_{f}\left(r, D_{j}\right)}{\operatorname{deg} D_{j}} \leq 2 n T_{f}(r)+O(1)
$$

where $O(1)$ is a constant independent of $r$.

Proof. The proof is essentially the same as for the Main Theorem. The only difference is that now we are working simultaneously on the radii $r$ and $r^{-1}$. In the proof of the Main Theorem, we arranged $\left|G_{j} \circ f\right|_{r}$ in increasing order in (1). We may not be able to use the same index order for $\left|G_{j} \circ f\right|_{r^{-1}}$. Thus, in the worst case, we may need to use $2 n$ distinct proximity functions, and hence we get $2 n$ instead of $n$ on the right-hand side of the inequality.

For the First Main Theorem in this context, see [C, Theorem III.4.7].

\section{CONCLUding REMARKS}

The First Main Theorem and the fact that the proximity functions are always bounded below imply that if $f$ is a non-constant analytic curve not completely contained in a projective hypersurface $D$, then

$$
0 \leq \delta_{f}(D) \leq 1
$$

Moreover, $\delta_{f}(D)=1$ if the image of $f$ omits $D$. Thus, Corollary 11 implies that a non-Archimedean analytic curve in a projective variety $X$ can omit at most $\operatorname{dim} X$ very ample divisors in general position.

When $X=\mathbb{P}^{N}(\mathbf{K})$ and the $D_{j}$ are hyperplanes in general position, simple examples show that the main results here are optimal, even if $f$ is assumed linear non-degenerate; cf. CY. In the complex case, one conjectures; (cf. L Conjecture VII.5.1]) that for $X$ smooth, for $D_{j}$ smooth with at worst normal crossing singularities at their intersections, and for algebraically non-degenerate holomorphic curves, one need not divide by $\operatorname{deg} D_{j}$ on the left-hand side of the inequality, and in place of $\operatorname{dim} X$ on the right-hand side, one has the degree of the anti-canonical bundle with respect to an ample divisor. Note that when $X=\mathbb{P}^{N}(\mathbb{C})$, one has $N+1$, the degree of the anti-canonical bundle, rather than $N$, on the right-hand side of the optimal inequality.

In light of the above-cited conjecture over the complex numbers, it would also be interesting if one could prove an inequality involving the degree of the canonical bundle of $X$ (over characteristic zero ground fields) or where one does not need to divide by the degree of the hypersurfaces on the left-hand side.

It might also be interesting to develop a Nevanlinna theory from domains of the form $\mathbf{K} \backslash\left\{a_{1}, \ldots, a_{m}\right\}$, generalizing the $\mathbf{K} \backslash\{0\}$ case treated here. One might also want to distinguish between essential and non-essential singularities at each puncture.

Finally, it would be interesting to obtain an inequality of Second Main Theorem type for non-Archimedean analytic curves into arbitrary varieties that contains some sort of ramification term, such as by using truncated counting functions. We plan to address this in a future work. 


\section{REFERENCES}

[C] Cherry, W., Hyperbolic p-adic analytic spaces, Ph.D. thesis, Yale University, 1993.

[CY] Cherry, W. and YE, Z., Non-Archimedean Nevanlinaa theory in several variables and the non-Archimedean Nevanlinna inverse problem, Trans. Amer. Math. Soc. 349 (1997), 5043-5071. MR:1407485 (98c:11072)

[EF] Evertse, J.-H. and Ferretti R. G., A generalization of the Subspace Theorem with polynomials of higher degree, Preprint arXiv:math.NT/0408381.

[HY] Hu, P.-C. and YANG, C.-C., Meromorphic functions over non-Archimedean fields, Kluwer, 2000. MR.1794326 (2002a:11085)

[L] LANG, S., Number Theory III, Encyclopedia of Mathematical Sciences 60, Springer-Verlag, 1991. MR 1112552 (93a:11048)

[R] Ru, M., A note on p-adic nevanlinna theory, Proc. Amer. Math. Soc. 129 (2001), 12631269. MR.1712881 (2001h:11097)

[W] Van der Waerden, B. L., Algebra, Vol. 2, 7th ed., Springer-Verlag, New York, 1991. MR:1080173 (91h:00009b)

Institute of Mathematics, 18 Hoang Quoc Viet, Cau Giay, Hanoi, Vietnam

E-mail address: tthan@math.ac.vn 